\title{
Role of PEG 2000 in the surface modification and physicochemical characteristics of pyrazinamide loaded nanostructured lipid carriers
}

\author{
GOURAB KARMAKAR ${ }^{\mathrm{a}}$, PRASANT NAHAK ${ }^{\mathrm{a}}$, PRITAM GUHA ${ }^{\mathrm{a}}$, BIPLAB ROY ${ }^{\mathrm{a}}$, \\ RANENDU KUMAR NATH ${ }^{\mathrm{b}}$ and AMIYA KUMAR PANDA ${ }^{\mathrm{c}, *}$ \\ ${ }^{a}$ Department of Chemistry, University of North Bengal, Darjeeling, West Bengal 734 013, India \\ ${ }^{b}$ Department of Chemistry, Tripura University, Suryamaninagar, Tripura 799 022, India \\ ${ }^{\mathrm{c}}$ Department of Chemistry and Chemical Technology, Vidyasagar University, Midnapore, West Bengal 721 102, \\ India \\ E-mail: akpanda@mail.vidyasagar.ac.in
}

MS received 13 January 2018; revised 12 March 2018; accepted 12 March 2018; published online 29 March 2018

\begin{abstract}
Hydrogenated soy phosphatidylcholine, tristearin and oleic acid were employed in preparing nanostructured lipid carriers (NLC). Surface modified NLCs ( NLC $_{\mathrm{PEG}}$ ) were formulated by adding polyethylene glycol 2000 (PEG 2000) in the dispersion medium along with Tween 60. Hot homogenization followed by ultrasonication technique was used as the preparative procedure. $0.01 \%(\mathrm{w} / \mathrm{v}, \mathrm{g} / \mathrm{mL})$ PEG 2000 was found to be the saturation limit for the studied formulations. Pyrazinamide (PYZ) was incorporated in NLC; the base and drug-loaded formulations were characterized by combined dynamic light scattering (DLS), differential scanning calorimetry (DSC), transmission electron microscopy (TEM) and atomic force microscopy (AFM). NLC $C_{\mathrm{PEG}}$ was more stable than the conventional NLC formulations. Added PEG 2000 provided extra steric stability to NLC $_{\text {PEG }}$ systems by introducing an additional layer over their surface. The presence of the additional layer of PEG 2000 also offered a preventative barrier towards the expulsion of surface accumulated PYZ. Considerable improvement in entrapment efficiency (EE\%), drug loading (DL\%) and desirable sustained release profile could be achieved in NLC.
\end{abstract}

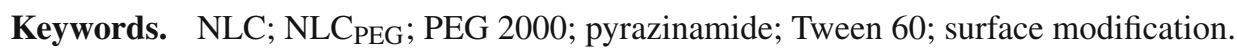

\section{Introduction}

Nanocolloidal lipid-based drug delivery systems have enormous possibilities in the improvement of pharmacodynamics and pharmacokinetics of a large number of active pharmaceutics. ${ }^{1-5}$ Some well-known commonly used drug delivery systems are a microemulsion, liposomes, nano suspensions, polymeric nanoparticles, etc. However, almost all the mentioned lipid-based drug delivery systems suffer from serious limitations like low drug incorporation, fast release of incorporated drugs, low affinity towards hydrophilic drug, biocompatibility, etc. ${ }^{6-10}$ These common disadvantages of the lipid-based drug delivery systems can successfully be overcome in nanostructured lipid carriers (NLC). NLCs are the modified form of solid lipid nanoparticles, also known

\footnotetext{
*For correspondence
}

as the second generation solid lipid nanoparticles. ${ }^{1-5} \mathrm{~A}$ blend of two or more structurally dissimilar biocompatible lipids (solid or liquid) is used in the preparation of NLC. The created structural imperfections by the sterically different lipid molecules can effectively host the pharmaceutics to a considerable extent and effectively reduce the drug loss by unwanted drug leakage. NLC systems thus effectively can overcome all major limitations of the conventional drug delivery systems and prove as the most promising agents. Although NLCs are advantageous, but problems like lipid modification, high rate of coagulation, separation of the lipid phase, poor incorporation of the hydrophilic drug, etc., have restricted its pertinence and market availability. ${ }^{1-3,11-13}$ These shortcomings are the serious challenges to a pharmacist in developing NLCs as novel drug delivery agent.

Several attempts have been made previously to enhance the stability and performance of NLC by 
employing different combinations of surfactants and polymers in the dispersion medium. ${ }^{9,14,15}$ In the case of the surfactant-polymer stabilized NLCs, nonionic surfactants and polymers provide better performance due to their biocompatibility and nontoxicity. ${ }^{9}$ Moreover, the nonionic polymers provide a great deal of steric stability and effectively reduce lipid modification during storage that is directly related to the coagulation rate of NLC. It has been established that nonionic polymers produce a layer over the NLC surface and effectively reduce the coagulation. ${ }^{9,16}$ The polymer layer is also effective in reducing the easy escape of the surface accumulated hydrophilic drug components. But a detailed study regarding the effect of nonionic polymers on the stability of NLC and the exact role of the polymer layer on the drug incorporation/release mechanism is still lagging in the literature. To uncover those facts, different concentrations of a nonionic biocompatible polymer PEG 2000 have been used in combination with the nonionic surfactant Tween-60 in developing surface-modified NLC $\mathrm{PEG}_{\mathrm{PEG}}$ systems and subjected to the delivery of a hydrophilic drug Pyrazinamide, PYZ. ${ }^{6-9}$

PYZ is a well-known first line drug in the treatment of active tuberculosis. Generally, PYZ is used in combination with isoniazide and rifampicin in the treatment of active tuberculosis. ${ }^{17-20}$ It is also used as a potent drug for ueicosuric disuses. In addition to this, hypouricemia and hyperuricosuria have been effectively diagnosed using PYZ. ${ }^{18,19}$ But higher aqueous solubility, high rate of excretion and high dose frequency have restricted the pharmaceutical use of PYZ, ${ }^{18,19}$ thus warranting a suitable vehicle for delivery. A number of attempts have been made to develop suitable solid lipid-based drug delivery systems to overcome the limitations. ${ }^{17-20}$ Most of the proposed formulations suffer from the limitations like low entrapment efficiency, drug loading capacity and faster release of incorporated PYZ. These have motivated the present work to develop suitable NLC system for PYZ and to improve the performance through surface modification using nonionic polymer PEG 2000. No such prior attempts have been made in developing surface-modified NLC $_{\mathrm{PEG}}$ for the delivery of PYZ to the best of our knowledge.

In the present study, surface-modified $\mathrm{NLC}_{\mathrm{PEG}}$ formulations were prepared; stability and efficiency as a delivery agent of hydrophilic pharmaceutics PYZ were compared with the conventional NLC without having any surface modifications provided by nonionic polymer PEG 2000. Conventional NLC and surface modified $\mathrm{NLC}_{\mathrm{PEG}}$ formulations were prepared using the hot homogenization followed by ultrasonication method. The obtained formulations were subjected to physicochemical characterization using DLS, DSC, TEM and
AFM. Water-soluble PYZ was used as a drug and PYZ-loaded formulations were characterized using the mentioned analytical techniques to gather information regarding the location of the incorporated PYZ. Conventional NLC and $\mathrm{NLC}_{\mathrm{PEG}}$ formulations were further characterized by the evaluation of drug loading and entrapment efficiency (DL\% and EE\%). The release of the incorporated PYZ was also studied to evaluate the potentiality of the studied formulations as a delivery system for PYZ. The obtained release profiles were further analyzed using different release models for the evaluation of the exact release mechanism of the incorporated PYZ. Such comprehensive set of work can be helpful in providing a concept in the development of different surface-modified NLC formulations for water-soluble PYZ and other drugs of this family.

\section{Material and methods}

\subsection{Materials}

Hydrogenated soy phosphatidylcholine (HSPC), tristearin (TS) and oleic acid (OA) were purchased from Sigma-Aldrich Chemicals (USA). Tween-60 and polyethylene glycol-2000 (PEG 2000) were procured from SRL, India. Pyrazinamide (PYZ) was obtained from Merck Specialties Pvt. Ltd, India. Solvents of analytical grade have been used throughout the study. Double distilled water having a molar conductivity of $2 \mathrm{mS} \mathrm{cm}^{-1}$ at $25^{\circ} \mathrm{C}$ was used for all the experiments.

\subsection{Preparation of $N L C$ and $N L C_{\mathrm{PEG}}$}

Conventional hot homogenization followed by ultrasonication was used for the preparation of the studied formulations. Details regarding the preparative procedure can be obtained in our recent publications. ${ }^{6-9} \mathrm{HSPC}$, TS and OA were used in the molar ratio 2:2:1 and the concentration of the studied formulations were maintained at $1 \mathrm{mM}$. In case of the NLC $\mathrm{CEG}_{\mathrm{PEG}}$ formulation, PEG 2000 was introduced by dissolving it in the dispersion medium. $0.0001,0.001,0.01$ and $0.1 \%(\mathrm{w} / \mathrm{v}, \mathrm{g} / \mathrm{mL})$ of PEG 2000 was used separately in the dispersion medium. $2 \mathrm{mM}$ aqueous Tween-60 solution was used as a dispersion medium for the studied formulations. During the preparation of PYZ-loaded NLCs, PYZ was introduced in the lipid physical mixture. In this study, concentration of PYZ was fixed at $10 \mu \mathrm{M}$ for the conventional NLC and NLC $\mathrm{PEG}_{\mathrm{PEG}}$ formulations.

\subsection{Instrumentation}

Hydrodynamic diameter $\left(\mathrm{d}_{\mathrm{h}}\right)$, polydispersity index (PDI) and zeta potential (Z.P.) of the NLCs were determined using dynamic light scattering technique (Zetasizer Nano ZS90 ZEN3690, Malvern Instruments Ltd., U.K.). Morphology of the prepared NLC and NLC PEG $_{\text {systems were studied }}$ 
using conventional TEM (Hitachi H-600, Japan) and AFM (Bruker Nanoscope V Multimode SPM). Thermal behavior of the studied formulations was analyzed using a differential scanning calorimeter (DSC, Mettler Toledo, Switzerland). Obtained thermal data were further analyzed using DSC1 $\mathrm{STAR}^{e}$ software for the evaluation of different thermodynamic parameters.

\subsection{Entrapment efficiency (EE\%) and drug loading capacity (DL\%)}

Method of centrifugation was used for the evaluation of EE\%

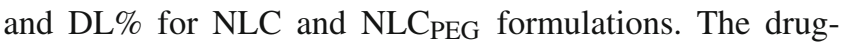

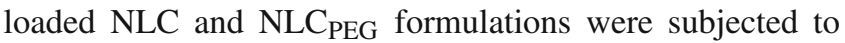
high-speed centrifugation at $15000 \mathrm{rpm}$ and at $4{ }^{\circ} \mathrm{C}$. Collected supernatant of the centrifuged samples were analyzed colorimetrically to determine the amount of free drug. In case of the colorimetric analysis, absorbance values of PYZ at different known concentrations in aqueous Tween 60 solution (2 $\mathrm{mM}$ ) were recorded and a standard absorbance vs. PYZ concentration graph was obtained. The standard graph was employed to determine the concentration of PYZ present in the obtained absorbance value of the collected supernatant of the centrifuged samples. EE\% and DL\% of the studied formulations were determined using the following equations: ${ }^{6-9}$

$$
\begin{aligned}
& E E \%=\frac{W_{\text {loaded } P Y Z}}{W_{\text {totaal } P Y Z}} \times 100 \% \\
& D L \%=\frac{W_{\text {loaded } P Y Z}}{W_{\text {lipid }}} \times 100 \%
\end{aligned}
$$

Where, $W_{\text {total PYZ }}, W_{\text {lipid }}$ and $W_{\text {loaded PYZ }}$ represent the weight of total PYZ added to the formulations, lipids used in NLC (or

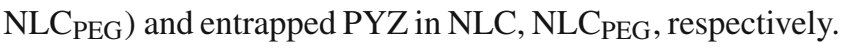

\subsection{In vitro release study}

Release kinetics of the incorporated PYZ from NLCs were studied using conventional dialysis bag approach $(12 \mathrm{kD}){ }^{6-9}$

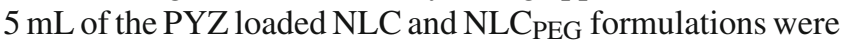
taken inside the dialysis bag and immersed into $20 \mathrm{~mL}$ of release medium. $2 \mathrm{mM}$ aqueous Tween- 60 solution was used as the release medium in the present study. The release experiment was carried out by maintaining sink condition under constant stirring. The released drug was quantified colorimetrically.

\section{Results and Discussion}

\subsection{Solution phase behavior of $N L C$ and $N L C_{\mathrm{PEG}}$}

Hydrodynamic diameter $\left(\mathrm{d}_{\mathrm{h}}\right)$ is a marker indicative of the stability, loading capacity, entrapment efficiency and the release rate of the incorporated drug. ${ }^{2-4,7,8} \mathrm{~d}_{\mathrm{h}}$ was monitored with respect to time and presented graphically in the panel A of Figure 1. The size of the
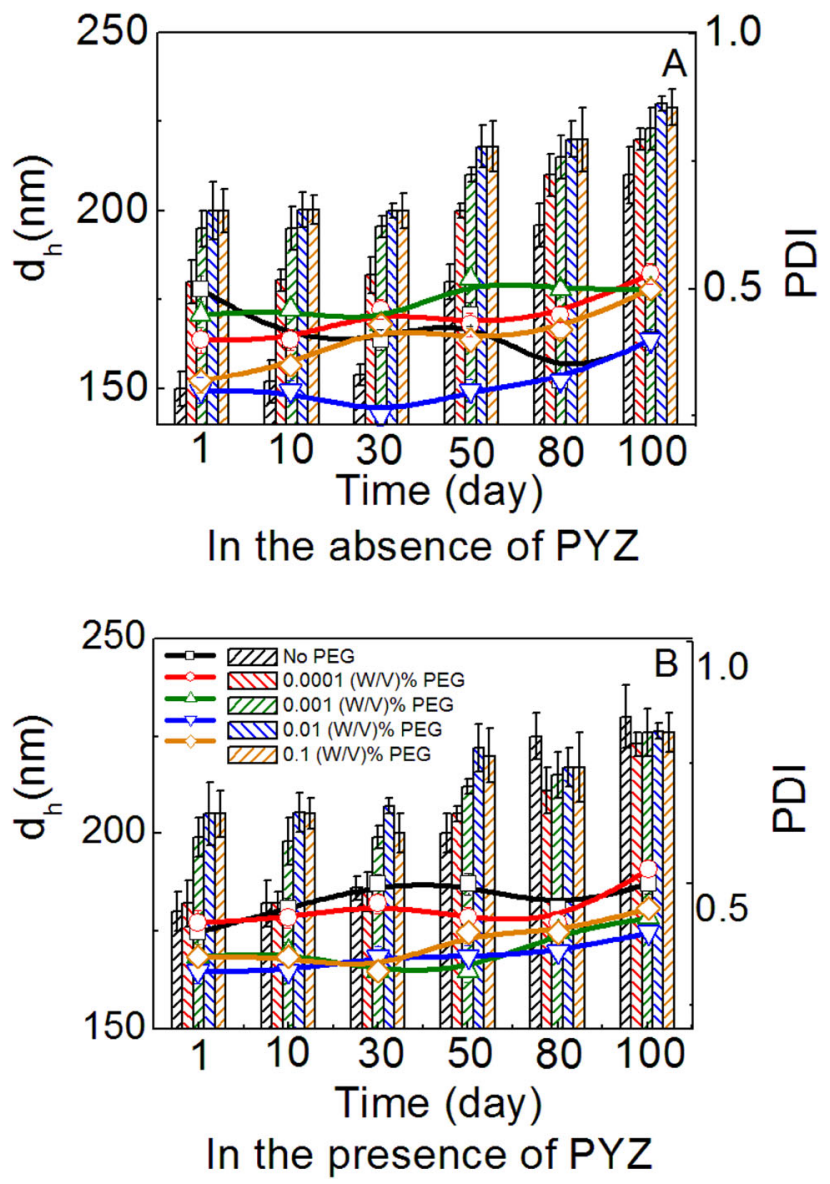

Figure 1. Effect of PEG 2000 concentration on the NLC hydrodynamic diameter $\left(\mathrm{d}_{\mathrm{h}}\right) /$ polydispersity index (PDI) $v s$. time profile in the presence (panel A) and absence of PYZ (panel B) at $25{ }^{\circ} \mathrm{C}$. HSPC:TS:OA = 2:2:1. Concentration of PEG 2000 are mentioned inside the figure.

conventional NLC varied in the range of 150-210 nm. PEG 2000 coated formulations were in the range of 200 $230 \mathrm{~nm}$, higher than the conventional NLC, due to the presence of an additional layer of the added PEG 2000. There occurred a progressive increase in the $d_{h}$ values with increasing PEG 2000 concentration up to 0.01 (w/v, $\mathrm{g} / \mathrm{mL}$ ) PEG 2000, beyond which further increase in PEG 2000 had no significant effect on the NLC size. This indicates the saturation point of PEG 2000 concentration; at this concentration, palisade layer of the NLC becomes saturated.

A clear idea regarding the solution phase stability during the storage can be obtained from the $d_{h}$ $v s$. time profile (panel A of Figure 1) for the studied formulations. The $\mathrm{d}_{\mathrm{h}}$ of the conventional NLC and $\mathrm{NLC}_{\mathrm{PEG}}$ formulations were monitored for 100 days and no significant fluctuation in $\mathrm{d}_{\mathrm{h}}$ value was observed. The observation indicated the formation of stable nanocolloidal suspension. ${ }^{1,2,21}$ In addition to this, a smooth progressive increment in the $d_{h}$ value was noted with 
the storage time for all the studied formulations. The coagulation of the nanocolloidal suspension was mainly responsible for the observed phenomenon. The observed growth rate of the $\mathrm{NLC}_{\mathrm{PEG}}$ formulations were found to be lower than the conventional NLC system. A reduction in growth rate indicated the reduced coagulation in the presence of additional PEG 2000 layer along with the non-ionic surfactant Tween 60. The additional layer of PEG 2000 provided extra steric stability to the NLC $_{\mathrm{PEG}}$ and restricted the coagulation phenomenon among the suspended lipid particles to a considerable extent.

$\mathrm{d}_{\mathrm{h}}$ vs. time profiles for PYZ loaded NLC and NLC $\mathrm{PEG}_{\mathrm{PE}}$ formulations were also presented in the panel $\mathrm{B}$ of Figure $1 . \mathrm{d}_{\mathrm{h}}$ of PYZ loaded conventional NLC and NLC $\mathrm{CEG}_{\mathrm{PE}}$ formulations were found in the range of 180-230 nm and $185-233 \mathrm{~nm}$, respectively. The observed enhancement in the $d_{h}$ value in case of the conventional NLC indicated the accumulation of PYZ on the surface and suggested a shell-enriched NLC formulation. But the observed size enhancement in the presence of PYZ in case of $\mathrm{NLC}_{\mathrm{PEG}}$ is not significant. The observed result indicated the accumulation of the incorporated PYZ in between the PEG 2000 layer and the lipid palisade layer of $\mathrm{NLC}_{\mathrm{PEG}}$ formulation.

PDI of the nanocolloidal drug delivery systems is another crucial parameter because it regulates the homogeneity of nanocolloidal suspensions. The observed PDI values (shown in the panel A of Figure 1) of the studied formulations were in the range 0.3-0.5 indicative of substantially homogeneous formulations. Observed fluctuation in the PDI vs. time profile suggests ongoing crystalline modification in the NLC core. Crystalline modifications make the lipid core of the NLCs more perturbed, hence the homogeneity of the formulations decreases during storage. In the presence of PEG 2000, fluctuation in the PDI values were considerably less which suggests stability enhancement compared to the conventional NLC. In case of PYZ-loaded NLC formulation (panel B of Figure 1), a small increase in the PDI indicates reduction in the surface homogeneity due to the accumulation of PYZ on the palisade layer of NLC. On the other hand, insignificant variation in PDI for the PYZ-loaded $\mathrm{NLC}_{\mathrm{PEG}}$, compared to the corresponding base system, indicates the accumulation of PYZ in between the lipid palisade layer and the additional polymer layer. Due to the penetration of the incorporated PYZ into the PEG 2000 layer, no surface irregularity was noted at the surface of PYZ-loaded NLC $_{\mathrm{PEG}}$ formulations.

Zeta potential (Z.P.) is directly related to the surface charge and electrostatic stability of colloidal particles. ${ }^{2-4,7,8}$ Z.P. of the conventional NLC formulation was in the range of -22 to $-24 \mathrm{mV}$ (panel A of Figure 2),

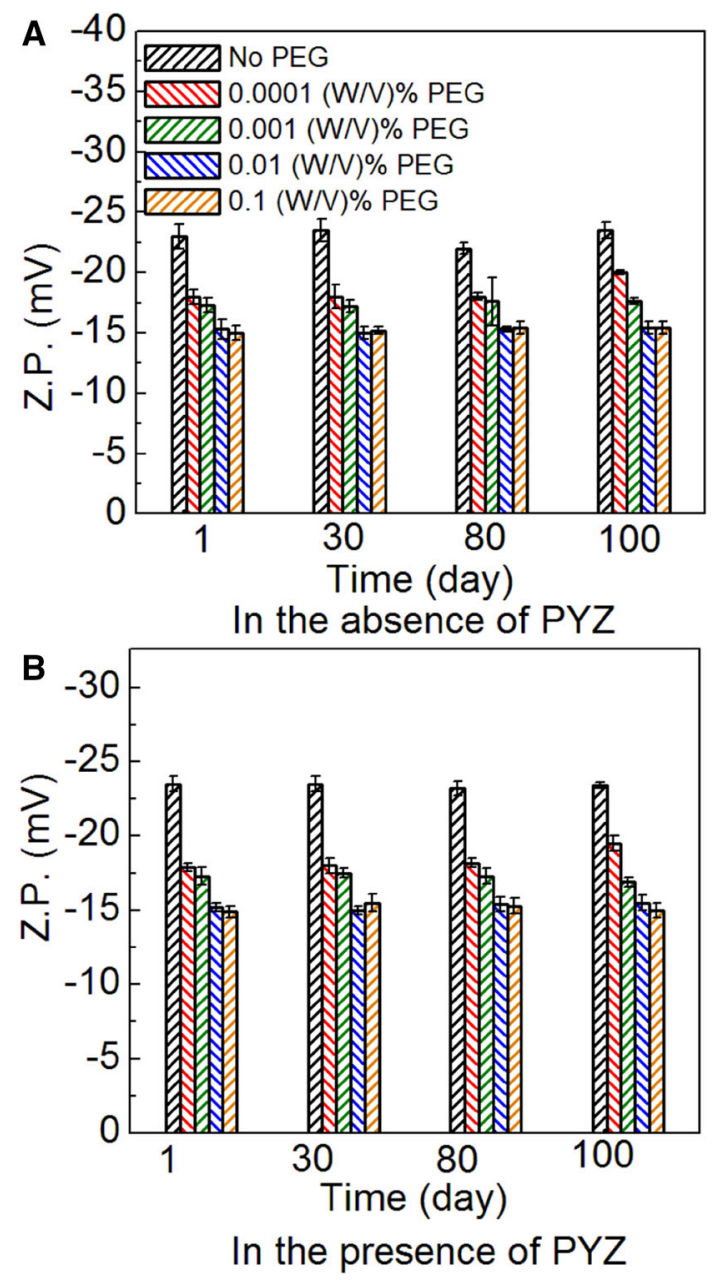

Figure 2. Effect of PEG 2000 concentration on the zeta potential (Z.P.) of NLC vs. time profile in the presence (panel A) and absence of PYZ (panel B) at $25^{\circ} \mathrm{C}$. HSPC:TS:OA = 2:2:1. Concentration of PEG 2000 is mentioned in the figure.

which was reduced to -15 to $-20 \mathrm{mV}$ in presence of PEG 2000. Reduction in the negative magnitude of Z.P. was due to the pressure of hydrophilic polymer layer (PEG-2000) that effectively masked the surface change of Z.P. of NLC. With increasing concentration of PEG 2000 , magnitude of the zeta potential value decreased till the concentration of PEG 2000 was at $0.01 \%$ (w/v, $\mathrm{g} / \mathrm{mL}$ ), after which an increase in PEG 2000 concentration exhibited no significant influence. Increasing PEG concentration increased the thickness of polymer layer over the NLC surface and progressively reduced the surface charge. In the case of PYZ-loaded NLCs (both in the absence and presence of PEG 2000) no significant influence on the Z.P. value was noted (panel B of Figure 2). Insignificant impact of time on the Z.P. of the base and drug-loaded formulations also signify considerable solution phase stability for the formulations under experiment. The observed fluctuation in the $d_{h}$ 


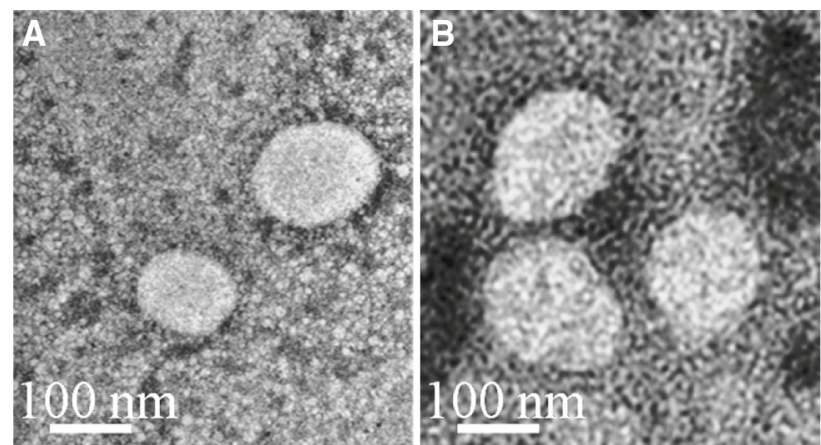

Figure 3. TEM images of NLC (HSPC:TS:OA, 2:2:1) having $0.01 \%(\mathrm{w} / \mathrm{v}, \mathrm{g} / \mathrm{mL})$ of PEG 2000 in the absence $(\mathrm{A})$ and presence (B) of PYZ. Scale bars are shown inside the images.

with respect to time was due to the inside lipidic modification in the core of NLC and $\mathrm{NLC}_{\mathrm{PEG}}$ formulations. On the other hand, Z.P. is related to the surface charge of the studied formulations. No significant change in the Z.P. value with respect to the storage time clearly indicated that the lipidic modification has no significant influence on the surface of the studied formulations.

\subsection{Morphological studies}

Shape and surface morphology of the NLCs in the absence and presence of PEG were analyzed by combined TEM and AFM studies. Spherical morphology with a smooth surface was observed through the TEM measurements as shown in the panels A and B of Figure 3.

A small reduction in size in the case of TEM studies indicates the loss of the hydration sphere associated with the NLC and $\mathrm{NLC}_{\mathrm{PEG}}$ during the drying process of sample preparation. ${ }^{6-8}$ The loss of hydration sphere caused size constriction of the NLCs. Morphology of the studied formulations was further analyzed through the AFM measurements. Representative AFM image of $\mathrm{NLC}_{\mathrm{PEG}}$ formulation is shown in Figure 4 along with the three-dimensional overview and height analysis profile. The observed results were in agreement with the DLS and TEM studies. The height of the NLCs was in the range of 40-60 nm. Significant reduction in height can be explained by the collapse of NLC during the vacuum drying process. ${ }^{22,23}$ The presence of additional polymer layer could not be clearly identified through the TEM and AFM analyses. During the loss of hydration sphere, the layer of PEG 2000 also got collapsed. More sophisticated morphological analysis like FF-TEM and/or cryo-TEM studies are considered to be helpful in obtaining the impact of the polymer layer, considered to be the future perspectives.
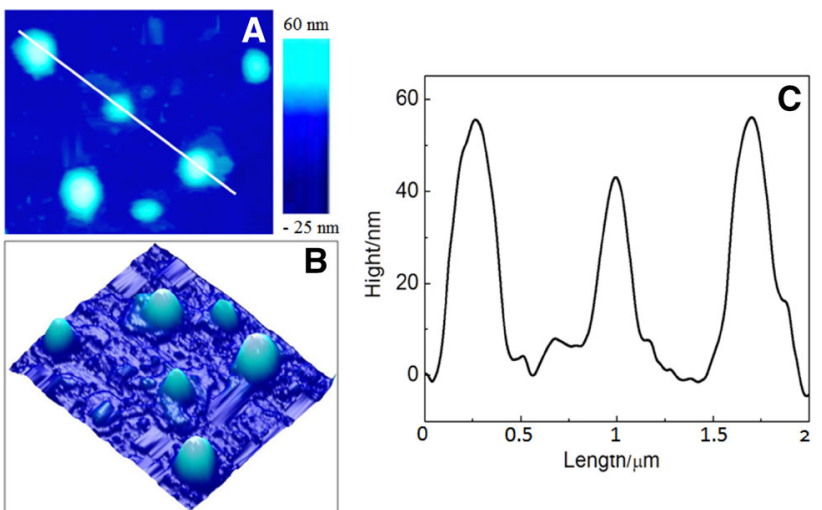

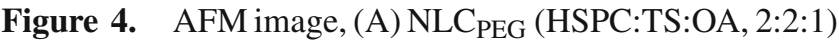
having $0.01 \%(\mathrm{w} / \mathrm{v}, \mathrm{g} / \mathrm{mL})$ of PEG 2000. Panels (B) and (C) represent three-dimensional surface morphology and corresponding height analysis, respectively.

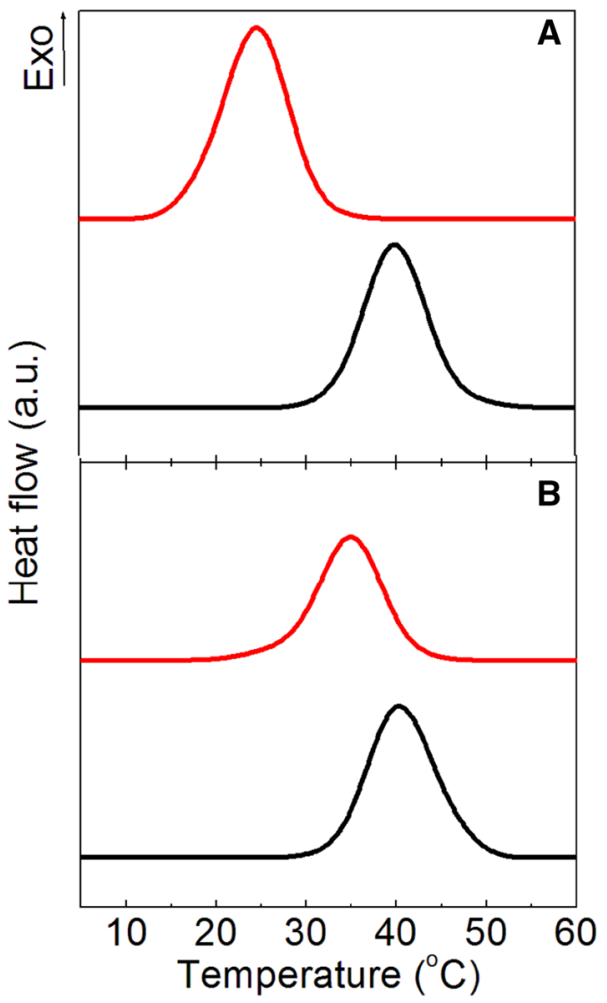

Figure 5. DSC cooling thermogram

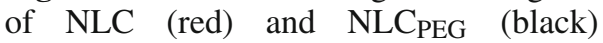
(HSPC:TS:OA, 2:2:1) having $0.01 \%$ (w/v, g/mL) of PEG 2000 in the absence (panel A) and presence (panel B) of PYZ. Scan rate: $2{ }^{\circ} \mathrm{C} \min ^{-1}$.

\subsection{Differential scanning calorimetric studies}

DSC is a very useful technique to collect the information regarding internal morphology and crystallinity of NLCs. ${ }^{6,9,16}$ Representative DSC thermogram of the conventional NLC in the absence and presence of PEG 2000 are shown in Figure 5. In both the cases, only a single prominent peak appeared that indicates negligible 
Table 1. Phase transition temperature $\left(\mathrm{T}_{\mathrm{m}}\right)$, half-peak width $\left(\Delta \mathrm{T}_{1 / 2}\right)$, change in enthalpy $(\Delta \mathrm{H})$ and heat capacity $\left(\Delta \mathrm{C}_{\mathrm{p}}\right)$ of the base and PYZ loaded NLC (HSPC:TS:OA, 2:2:1) having different concentrations of PEG 2000.

\begin{tabular}{|c|c|c|c|c|c|}
\hline & $\%$ PEG $2000(\mathrm{w} / \mathrm{v}, \mathrm{g} / \mathrm{mL})$ & $\mathrm{T}_{\mathrm{m}}\left({ }^{\circ} \mathrm{C}\right)$ & $\Delta \mathrm{T}_{1 / 2}\left({ }^{\circ} \mathrm{C}\right)$ & $\Delta \mathrm{H}\left({\left.\mathrm{kcal} . \mathrm{mol}^{-1}\right)}^{-1}\right.$ & $\Delta \mathrm{C}_{\mathrm{p}}\left(\mathrm{kcal} . \mathrm{mol}^{-1} \mathrm{C}^{-1}\right)$ \\
\hline \multirow[t]{5}{*}{ Bare NLC } & 0 & 27 & 8.5 & 32 & 3.76 \\
\hline & 0.0001 & 32 & 8.3 & 32.9 & 3.96 \\
\hline & 0.001 & 37 & 9.2 & 33 & 3.58 \\
\hline & 0.01 & 40 & 9 & 32.5 & 3.61 \\
\hline & 0.1 & 40.1 & 9.2 & 33.4 & 3.63 \\
\hline \multirow[t]{5}{*}{ PYZ-loaded NLC } & 0 & 35 & 9.5 & 33.1 & 3.48 \\
\hline & 0.0001 & 31.3 & 9.5 & 32.5 & 3.42 \\
\hline & 0.001 & 36.2 & 9 & 32.3 & 3.58 \\
\hline & 0.01 & 39.2 & 8 & 33.2 & 4.15 \\
\hline & 0.1 & 39.3 & 9.3 & 33.3 & 3.58 \\
\hline
\end{tabular}

Concentration of the NLC:1 mM. All the DSC studies were performed on day 1 of the NLC preparation. Scan rate: $2{ }^{\circ} \mathrm{C} \min ^{-1}$

lipidic modification inside NLC by the PEG. ${ }^{6,9,16}$ Phase transition temperature $\left(\mathrm{T}_{\mathrm{m}}\right)$ varied in the range 28-35 (in the absence of PEG 2000) and $32-40.1{ }^{\circ} \mathrm{C}$ (in the presence of PEG 2000), respectively. $T_{m}$ value noted was substantially reduced for both the types of NLCs in comparison to the bulk lipid mixture. The observed reduction can be explained on the basis of structural reorganization and subsequent generation of structural imperfections during the formation of NLC and $\mathrm{NLC}_{\mathrm{PEG}}$. In addition to this, a significant reduction in size is also an important cause for the reduction in $\mathrm{T}_{\mathrm{m}}$ value for NLC and $\mathrm{NLC}_{\mathrm{PEG}}$ systems. ${ }^{6-8}$ The observed cooling thermogram was more prominent than the heating thermogram. Downshift in the $T_{m}$ value in the cooling thermogram in comparison to the heating thermogram indicates the liquid crystalline-like behavior of the NLCs. ${ }^{24-27}$ Due to its prominence, the cooling thermograms were further analyzed to evaluate the different thermodynamic parameters, as summarized in Table 1.

$\mathrm{T}_{\mathrm{m}}$ value of the NLC was $28{ }^{\circ} \mathrm{C}$ which varied in the range of $32-40.1{ }^{\circ} \mathrm{C}$ in the presence of PEG 2000. An increase in the $T_{m}$ value indicates the existence of an additional layer of PEG 2000 over the NLC surface that retarded the phase transition. With an increase in the concentration of PEG 2000, an enhancement in $\mathrm{T}_{\mathrm{m}}$ indicates the formation of a thicker layer of PEG 2000 upto the saturation point $(0.01 \%)$. Insignificant difference in the $\Delta \mathrm{H}$ and $\Delta \mathrm{C}_{\mathrm{p}}$ for the NLCs without and with PEG 2000 indicates that the added PEG 2000 forms an outer layer over the NLC surface and does not have any significant interaction with the internal morphology of the NLC. Also, no observable change in $\Delta \mathrm{T}_{1 / 2}$ was recorded upon the addition of PEG 2000 to the NLC.

Panel B of Figure 5 shows the DSC thermograms of PYZ-loaded NLCs. $T_{m}$ value of the PYZ loaded NLC was higher than the bare NLC. Enhanced $\mathrm{T}_{\mathrm{m}}$ value upon
PYZ inclusion clearly indicates the formation of a shellenriched NLC. The presence of the hydrophilic lipid head groups at the surface provided a dipolar attraction to the hydrophilic drug PYZ and increased its surface availability. On the other hand, a slight reduction in $T_{m}$ for PYZ-loaded $\mathrm{NLC}_{\mathrm{PEG}}$ indicates the penetration of incorporated PYZ in between the PEG 2000 layer and NLC surface. The presence of PYZ creates an irregularity in the molecular arrangement of PEG 2000 layer which subsequently reduces the shielding to some extent and a reduction in the $\mathrm{T}_{\mathrm{m}}$ was observed. PYZ did not impart any observable change in the $\Delta \mathrm{H}, \Delta \mathrm{C}_{\mathrm{P}}$ and $\Delta \mathrm{T}_{1 / 2}$ values for an obvious reason. Hydrophilic nature of the PYZ restricted its petitioning into the lipid core of NLC. Results further confirm the surface accumulation of PYZ and formation of a shell-enriched NLC system.

\subsection{Entrapment efficiency (EE) and drug loading (DL) capacity studies}

$\mathrm{EE} \%$ and DL\% of NLCs in the absence and presence of varying concentration of PEG 2000 were determined (Figure. 6). EE\% and DL\% were found to be $30 \%$ and $2.4 \%$, respectively, in the absence of PEG 2000, which are less than the previously reported NLCs loaded with amphiphilic drug molecules. PYZ is a hydrophilic drug. Due to its hydrophilic character, physical exclusion rate of the surface adsorbed PYZ is very high. Only a weak dipolar attraction between the amine group of PYZ and the hydrophilic lipid head groups (mainly the carboxylic acid groups of the OA molecule) present at the NLC surface hold the PYZ molecules. Considerable improvement in the EE\% and DL\% to $42-58 \%$ and $3.3-4.6 \%$, respectively, in the presence of PEG can be explained on the basis of an additional layer of PEG 2000 over 


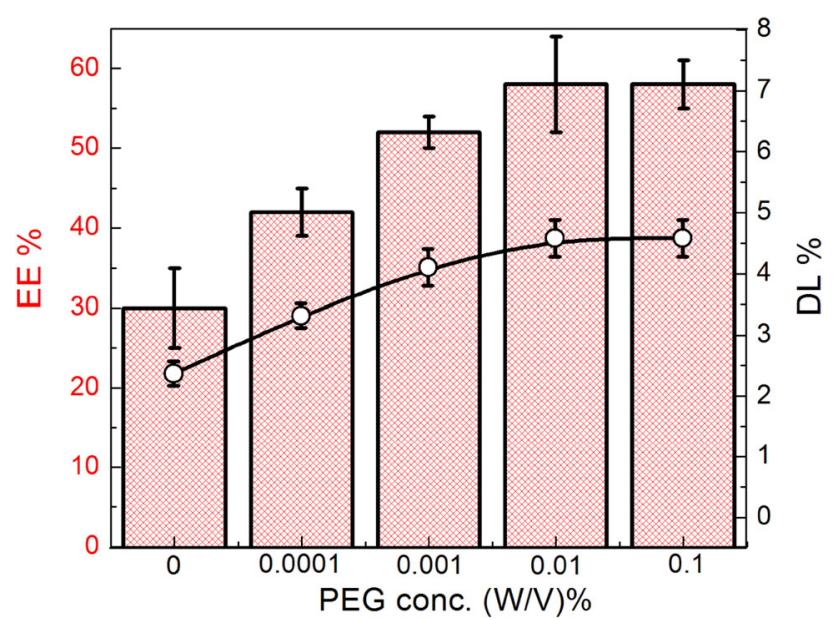

Figure 6. Variation in EE\% (bar) and DL\% (line) of NLC $_{\text {PEG }}$ (HSPC:TS:OA, 2:2:1) formulations with PEG 2000 concentration.

the NLC surface. In the presence of PEG, the incorporated drug got accumulated on the surface as well as in between the lipid palisade layer and the polymer layer. In addition to this, the PEG 2000 layer prevents easy physical exclusion of the incorporated drug. EE\% and DL\% of the NLC formulations also depended on the concentration of added PEG 2000; both the parameters increased with increasing the concentration of PEG 2000 up to $0.01 \% \mathrm{w} / \mathrm{v}$ after which no further enhancement was noted. With increasing concentration of PEG 2000, the shielding towards the physical exclusion of the PYZ increases due to the progressive increment in the thickness of the polymer layer. The thickness was found to increase till the attainment of saturation point.

\subsection{Kinetics of the drug release}

The release of the incorporated PYZ from the conventional NLC as well as NLC $\mathrm{PEG}_{\mathrm{PEG}}$ formulations was studied and compared. NLC with $0.01 \%$ PEG 2000 was considered for the release study for an obvious reason. The obtained release profile and the simple diffusion of PYZ from dialysis membrane have been graphically shown in Figure 7.

The simple diffusion of PYZ was taken as control for the release study to eliminate the effect of dialysis membrane over the release of PYZ. The release of PYZ from different NLCs in the absence and presence of PEG 2000 were found to be sustained. The release of PYZ from the NLC was monitored up to $48 \mathrm{~h}$ whereby a maximum of $84 \%$ PYZ got released from the conventional NLC which was further reduced to $61.3 \%$ in the case of added PEG 2000. In all the cases, a biphasic release pattern was noted: ${ }^{6,8,9}$ an initial burst release and then a

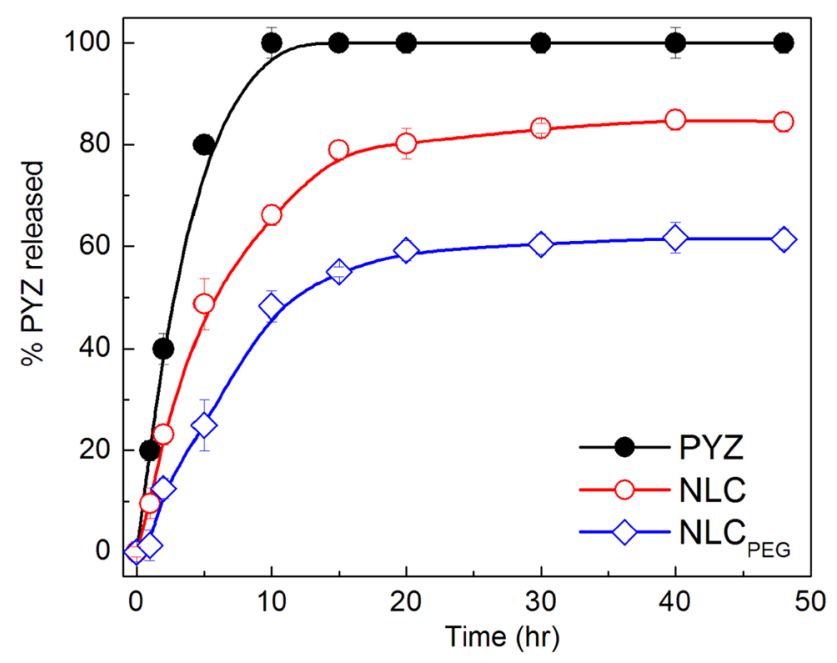

Figure 7. Release profiles of the free PYZ and PYZ from

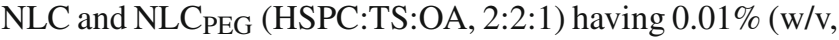
$\mathrm{g} / \mathrm{mL})$ of PEG 2000 at $25^{\circ} \mathrm{C}$.

sustained release of PYZ. In the case of the conventional NLC, $58 \%$ of the incorporated PYZ gets released during the initial burst within $8 \mathrm{~h}$, whereas only $36.3 \%$ of the incorporated PYZ got expelled as the initial burst release in the case of added PEG 2000. Results suggest that PEG provides better control and sustenance to the NLC in terms of the release of incorporated PYZ. An additional polymer layer over the NLC surface increases the micro-viscosity that effectively slows down the release of surface adsorbed PYZ.

The obtained release profiles were further fitted into different well-established release models to get a proper understanding regarding the drug release mechanism. DD solver 1.0, an add-in program, was used to obtain the dissolution data for the studied release profiles. ${ }^{28,29}$ In the present study, first order, zero order, Higuchi, Korsmeyer-Peppas and Weibull models were explored; the data have been presented in Table S1 in Supplementary Information. Obtained regression values favor the release of PYZ to follow Korsmeyer-Peppas model. The release rate of PYZ from the NLC were 7.21 and $4.64 \mathrm{~h}^{-1}$ in the absence and presence of PEG 2000 respectively. Release exponent (n) for NLC and NLC $_{\text {PEG }}$ were also evaluated for Korsmeyer-Peppas release model and the values were found to be $\leq 0.5$. Results indicate that the release of PYZ from NLC and $\mathrm{NLC}_{\mathrm{PEG}}$ was controlled by classical Fick diffusion mechanism.

\section{Conclusions}

The focus of the present work is on the development and the physicochemical characterization of surfacemodified NLC which were designed for the delivery of 
the hydrophilic drug, PYZ. PEG 2000, a nonionic polymer, was used for the surface modification. HSPC, TS and $\mathrm{OA}$ in the molar ratio 2:2:1 were used for the formulation of NLC. $0.01 \%(\mathrm{w} / \mathrm{v}, \mathrm{g} / \mathrm{mL}$ ) PEG 2000 was found to be the optimum concentration for the studied NLCs in imparting stability. A significant reduction in lipid modification and growth rate indicated the improvement in solution phase stability of NLC induced by the PEG. An additional adsorbed layer of PEG 2000 enhanced the steric stability of NLC. PYZ was also successfully incorporated in NLC. A detailed physicochemical characterization of PYZ-loaded formulation indicates the formation of shell-enriched NLC. EE\% and DL\% of NLC formulations were improved. In addition, the release of PYZ from NLC was further sustained by the surface-adsorbed PEG 2000. Thus, NLC PEG $_{\text {sys- }}$ tems could be considered as superior to the conventional NLC for the delivery of hydrophilic drug like PYZ. However, for a complete understanding of the role of nonionic polymer on the stability and performance of NLC formulations, more sophisticated studies like XRD (small and wide angle), FF-TEM, cryo-TEM, etc., are warranted. The relevant in vitro and in vivo biological studies are also required to evaluate the potential of their application.

\section{Supplementary Information (SI)}

The supplementary information contains the kinetic data of the drug release profiles in different well-established drug release models along with their mathematical expressions. In addition to this, a representative heating-cooling DSC thermogram of NLC $\mathrm{PEG}_{\mathrm{P}}$ system has been provided. Supplementary Information is available at www.ias.ac.in/chemsci.

\section{Acknowledgements}

Authors sincerely acknowledge Department of Science and Technology, Government of India, New Delhi for the financial support as research grant (SR/S1/PC-32/2011) for this work.

\section{References}

1. Müller R H 2007 Lipid nanoparticles: recent advances Adv. Drug Deliv. Rev. 59375

2. Müller R H, Radtke M and Wissing S A 2002 Solid lipid nanoparticles (SLN) and nanostructured lipid carriers (NLC) in cosmetic and dermatological preparations Adv. Drug Deliv. Rev. $54 \mathrm{~S} 131$

3. Müller R H, Radtke M and Wissing S A 2002 Nanostructured lipid matrices for improved microencapsulation of drugs Int. J. Pharm. 242121

4. Müller R H, Rúhl D and Runge S A 1996 Biodegradation of solid lipid nanoparticles as a function of lipase incubation time Int. J. Pharm. 144115
5. Ranpise N S, Korabu S S and Ghodake V N 2014 Second generation lipid nanoparticles (NLC) as an oral drug carrier for delivery of lercanidipine hydrochloride Colloid Surface B 11681

6. Karmakar G, Nahak P, Guha P, Roy B, Chettri P, Sapkota M, Koirala S, Misono T, Torigoe K, Ghosh S and Panda A K 2016 Effects of Fatty Acids on the Interfacial and Solution Behavior of Mixed Lipidic Aggregates Called Solid Lipid Nanoparticles J. Oleo. Sci. 65419

7. Nahak P, Karmakar G, Chettri P, Roy B, Guha P, Besra S E, Soren A, Bykov A G, Akentiev A V, Noskov B A and Panda A K 2016 Influence of Lipid Core Material on Physicochemical Characteristics of an Ursolic AcidLoaded Nanostructured Lipid Carrier: An Attempt To Enhance Anticancer Activity Langmuir 329816

8. Nahak P, Karmakar G, Roy B, Guha P, Sapkota M, Koirala S, Chang C-H and Panda A K 2015 Physicochemical studies on local anaesthetic loaded second generation nanolipid carriers RSC Adv. 526061

9. Sapkota M, Karmakar G, Nahak P, Guha P, Roy B, Koirala S, Chettri P, Das K, Misono T, Torigoe K and Panda A K 2015 Effect of polymer charge on the formation and stability of anti-inflammatory drug loaded nanostructured lipid carriers: physicochemical approach RSC Adv. 565697

10. Li W, Das S, Ng K-y and Heng P W S 2011 Formulation, Biological and Pharmacokinetic Studies of Sucrose Ester-Stabilized Nanosuspensions of Oleanolic Acid Pharm. Res. 282020

11. Shah R M, Malherbe F, Eldridge D, Palombo E A and Harding I H 2014 Physicochemical characterization of solid lipid nanoparticles (SLNs) prepared by a novel microemulsion technique J. Colloid Interf. Sci. 428 286

12. Tamjidi F, Shahedi M, Varshosaz J and Nasirpour A 2013 Nanostructured lipid carriers (NLC): A potential delivery system for bioactive food molecules Innov. Food Sci. Emerg. Technol. 1929

13. Yadav N, Khatak S and Singh Sara U V 2013 Solid lipid nanoparticles: a review Int. J. Appl. Pharm. 58

14. Man D K W, Casettari L, Cespi M, Bonacucina G, Palmieri G F, Sze S C W Leung G P H, Lam J K W and Kwok P C L 2015 Oleanolic Acid Loaded PEGylated PLA and PLGA Nanoparticles with Enhanced Cytotoxic Activity against Cancer Cells Mol. Pharm. 122112

15. Tang S, Gao D, Zhao T, Zhou J and Zhao X 2013 An evaluation of the anti-tumor efficac of oleanolic acidloaded PEGylated liposomes Nanotechnology 241

16. Medina-O'Donnell M, Rivas F, Reyes-Zurita F J, Martinez A, Martin-Fonseca S, Garcia-Granados A, FerrerMartín R M, Lupianez J A and Parra A 2016 Semisynthesis and antiproliferative evaluation of PEGylated pentacyclic triterpenes Eur. J. Med. Chem. 11864

17. Das $S$ and Chaudhury A 2011 Recent Advances in Lipid Nanoparticle Formulations with Solid Matrix for Oral Drug Delivery AAPS Pharm. Sci. Tech. 1262

18. Mukherjee S, Ray S and Thakur R S 2009 Solid Lipid Nanoparticles: A Modern Formulation Approach in Drug Delivery System Ind. J. Pharm. Sci. 71349

19. Pandey R and Khuller G K 2005 Solid lipid particlebased inhalable sustained drug delivery system against experimental tuberculosis Tuberculosis $\mathbf{8 5} 227$ 
20. Pandey R, Sharma S and Khuller G K 2005 Oral solid lipid nanoparticle-based antitubercular chemotherapy Tuberculosis $\mathbf{8 5} 415$

21. Wong H, Li Y, Bendayan R, Rauth M and Wu X 2007 Solid Lipid Nanoparticles for Anti-tumor Drug Delivery (Hoboken: Taylor and Francis)

22. Muhlen A Z, Muhlen E Z, Niehus H and Mehnert W 1996 Atomic Force Microscopy Studies of Solid Lipid Nanoparticles Pharm. Res. 131411

23. Shahgaldian P, Quattrocchi L, Gualbert J R M, Coleman A W and Goreloff P 2003 AFM imaging of calixarene based solid lipid nanoparticles in gel matrices Eur. J. Pharm. Biopharm. 55107

24. Larsson K 1997 Cubosomes and hexosomes for drug delivery Proc. Int. Symp. Controlled Release Bioact. Mater. 24198
25. Spicer P 2005 Cubosome processing industrial nanoparticle technology development Chem. Eng. Res. Des. 83 1283

26. Spicer P T 2003 Cubosome formation via dilution: Kinetic effects and consumer product implications ACS Symp. Ser. 861346

27. Spicer P T 2005 Progress in liquid crystalline dispersions: cubosomes Curr. Opin. Colloid In. 10 274

28. Dash S, Murthy P N, Nath L and Chowdhury P 2010 Kinetic modeling on drug release from controlled drug delivery systems Acta Pol. Pharm. 67217

29. Zhang Y, Huo M, Zhou J, Zou A, Li W, Yao C and Xie S 2010 DDSolver: an add-in program for modeling and comparison of drug dissolution profiles AAPS J. 12 263 